\title{
La santé à tout prix - mais qui va payer?
}

\section{Jacques de Haller}

Dr med., président du Comité de forumsante.ch

\author{
Le financement du système de santé est dans l'impasse! Ses sources de finance- \\ ment touchent à leur limite, et la Suisse doit impérativement trouver de nouveaux \\ modèles pour payer les soins de sa population. Quels peuvent être ces nouveaux \\ modèles de financement, et quelles conséquences auront-ils pour les divers acteurs \\ et utilisateurs?
}

Avec des besoins qui augmentent et un taux de financement direct (out-of-pocket) parmi les plus hauts en Europe (cf. OCDE: http://dx.doi.org/10.1787/healthdata-en), le financement du système de santé suisse est dans une impasse - les assuré-es ne peuvent en majorité plus contribuer davantage, et les collectivités ne sont souvent guère mieux loties. Et il ne s'agit pas là d'une affirmation idéologique ou polémique, mais bien de souligner un problème qui ne peut que devenir explosif et pour lequel des solutions doivent être réfléchies sans attendre l'urgence, toujours mauvaise conseillère.

On peut bien incriminer les coûts, qui augmentent régulièrement chez nous comme partout dans le monde développé; mais on le sait, réduire ou même stabiliser les coûts est illusoire sans bouleverser tout notre système de santé ni en abandonner quelques-uns des principes essentiels - ce qui serait faux... et ce que la population n'accepterait pas.

Plusieurs éléments témoignent de la précarité croissante du modèle de financement actuel. La nécessité de plus en plus massive d'allégements de prime, par exemple, démontre l'absurdité d'une mécanique qui arrive à bout et souligne aussi combien le système actuel devient invivable: selon les cantons, jusqu'à un habitant sur deux (NW) doit recevoir un subside d'allégement de prime allant jusqu'à $6000 \mathrm{CHF} / \mathrm{an} /$ foyer (AR) (moyenne suisse en 2014: 3118 CHF/an, 27\% de la population) (OFAS, www.bsv.admin.ch/dokumentation/).
La démarche hésitante de notre politique de santé pointe aussi cette impasse dans laquelle nous sommes engagés: entre "concurrence régulée» et libre marché, on tente en zig-zag de contenir les coûts de la santé à défaut de savoir en assurer le financement à long terme, et cette incertitude explique sans doute en bonne partie l'incapacité à prendre des décisions efficaces.

Reste que les solutions ne sont pas évidentes: assurer le financement durable d'un système de santé dont le budget croît de plusieurs pourcents par année ne peut que signifier trouver davantage d'argent, ou alors limiter les prestations - on comprend bien que l'alternative laisse nos politiciens perplexes!

Fiscaliser davantage le budget de la santé, c'est-à-dire avoir recours davantage à l'impôt pour le financer au lieu des primes - que ce soit l'impôt direct, l'impôt indirect (TVA) ou un prélèvement sur le revenu comme pour d'autres assurances sociales -, est un épouvantail pour la majorité du Parlement fédéral actuel. Limiter le libre-accès aux soins, respectivement restreindre le catalogue des prestations remboursées, est un «no-go» pour la gauche, notamment parce que cela donne une trop grande place aux moyens financiers personnels dans la possibilité d'accéder aux soins, et est donc source d'injustice.

Ainsi, il paraît important et urgent de lancer une discussion ouverte sur ce sujet, une discussion libre qui permette, espérons-le, de trouver des solutions innovantes, justes et durables.

La certitude de pouvoir recevoir les soins nécessaires en cas de besoin, quelle que soit la situation socioéconomique dans laquelle on sera le moment venu, est un acquis essentiel du $\mathrm{XX}^{\mathrm{e}}$ siècle, dans le monde occidental, et un facteur de stabilité de nos pays aussi, on doit s'en souvenir. Cet acquis a un coût - qui va le payer, à l'avenir?
Le mardi 2 février à l'Hôtel Bellevue à Berne, forumsante.ch organise pour la $17^{\mathrm{e}}$ fois un journée de réflexion sur un thème d'actualité: "La santé à tout prix - mais qui va payer?» Des conférenciers suisses et internationaux de haut niveau traiteront des aspects économiques, sociopolitiques, médicaux et éthiques de cette question. forumsante.ch ne s'arrête pas à discuter ce qui est, mais s'attache bien davantage à envisager et à réfléchir l'avenir - on y expose, construit et échange des idées pointues et originales sur le futur. Site Internet (programme): www.forumsante.ch; inscriptions: forumsante[at]furrerhugi.ch 